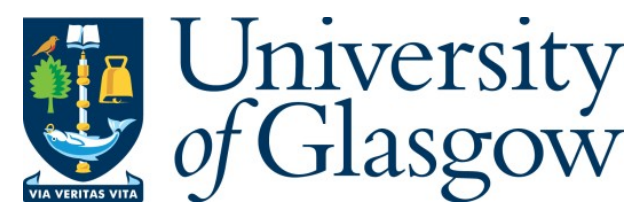

Griffiths, E., Fairlie-Clarke, K., Allen, J., Metcalf, C. J., and Graham, A. (2015) Bottom-up regulation of malaria population dynamics in mice co-infected with lung-migratory nematodes. Ecology Letters, 18(12), pp. 1387-1396.

There may be differences between this version and the published version. You are advised to consult the publisher's version if you wish to cite from it.

This is the peer reviewed version of the following article: Griffiths, E., Fairlie-Clarke, K., Allen, J., Metcalf, C. J., and Graham, A. (2015) Bottom-up regulation of malaria population dynamics in mice co-infected with lung-migratory nematodes. Ecology Letters, 18(12), pp. 1387-1396, which has been published in final form at http://dx.doi.org/10.1111/ele.12534. This article may be used for non-commercial purposes in accordance with Wiley Terms and Conditions for Self-Archiving.

http://eprints.gla.ac.uk/113103/

Deposited on: 7 January 2016

Enlighten - Research publications by members of the University of Glasgow http://eprints.gla.ac.uk 


\section{Bottom-up regulation of malaria population dynamics in mice co-infected with lung-migratory nematodes}

Emily C. Griffiths $\S^{1}$, Karen Fairlie-Clarke $\S^{2}$, Judith E. Allen ${ }^{3}$, C. Jessica E. Metcalf ${ }^{4,5}$, Andrea L. Graham ${ }^{* 4,5}$

${ }^{*}$ Corresponding author: algraham@princeton.edu

Email addresses of other authors: emilycgri@gmail.com

Karen.Fairlie-Clarke@glasgow.ac.uk

j.allen@ed.ac.uk and cmetcalf@princeton.edu

$\S$ These authors contributed equally to this work.

${ }^{1}$ National Disease Registers, Public Health England, 5 Old Fulwood Road, Sheffield, S10 3TG, UK

${ }^{2}$ Institute of Biodiversity, Animal Health \& Comparative Medicine, College of

Medical and Veterinary Life Sciences, University of Glasgow, Glasgow, G61

1QH, UK

${ }^{3}$ Institute of Immunology and Infection Research, School of Biological Sciences, University of Edinburgh, Edinburgh EH8 9YL, UK

${ }^{4}$ Department of Ecology and Evolutionary Biology, Princeton University, Princeton, NJ 08544, USA

${ }^{5}$ Fogarty International Center, National Institutes of Health, Bethesda MD 20892 USA

Statement of authorship: KF-C, JEA and ALG designed experiments and collected data. ECG and CJEM analysed data. ECG, KF-C, CJEM and ALG wrote the manuscript, and all authors contributed substantially to revisions.

Running title: Bottom-up regulation of malaria

Keywords:

Effective propagation

Parasite ecology

Community ecology

Target cell limitation

Resource regulation

Immune profile

Predator community

Co-infection

Type of Article: Letter

Abstract words: 143

Main text words: 4783

Tables: 1

Figures: 5

References: 46 


\section{Abstract}

When and how populations are regulated by bottom up versus top down processes, and how those processes are affected by co-occurring species, are poorly characterised across much of ecology. We are especially interested in the community ecology of parasites that must share a host. Here, we quantify how resources and immunity affect parasite propagation in experiments in near-replicate "mesocosms" - i.e., mice infected with malaria (Plasmodium chabaudi) and nematodes (Nippostrongylus brasiliensis). Nematodes suppressed immune responses against malaria, and yet malaria populations were smaller in co-infected hosts. Further analyses of within-host epidemiology revealed that nematode co-infection altered malaria propagation by suppressing target cell availability. This is the first demonstration that bottom-up resource regulation may have earlier and stronger effects than topdown immune mechanisms on within-host community dynamics. Our findings demonstrate the potential power of experimental ecology to disentangle mechanisms of population regulation in complex communities.

\section{Introduction}

Understanding the population dynamics of coexisting organisms has been a major challenge in ecology and evolution for over half a century (Andrewartha \& Birch, 1954). The diversity of drivers, importance of time-lagged effects, and influences of population structure mean that we are only beginning to quantify species interactions and predict their outcomes, even for classic, well-studied 
ecosystems. For example, analyses of decades of field data were needed to reveal that resource competition only transiently determines population growth rates in semi-arid grasslands (Martorell and Freckleton 2014) while external drivers such as weather exacerbate competition for island-dwelling (Coulson et al. 2001) and mountain-dwelling (Jacobson et al. 2004) ungulates. Feedbacks with predator abundance and specialisation may drive long-term oscillations in other systems (e.g., snowshoe hares; O'Donoghue et al. 1998). Increasingly, data capturing species dynamics across broad temporal and geographic scales have strengthened our ability to identify the core drivers of population dynamics (e.g., resource changes driving reduced amplitude of vole population oscillations across Europe; Cornulier et al. 2013), but covariance of key drivers across locations and time raises inferential challenges.

A multitude of ecological processes shape parasite population dynamics within hosts, so inferring species interactions in parasite communities from observational data is likewise challenging (Fenton et al. 2014). For example, parasite populations within hosts are regulated by the intrinsic reproductive rate of the parasites, top-down processes involving immunity, and bottom-up processes involving resources (Haydon et al. 2003, Pedersen and Fenton 2007). These processes are likely to drive the peaks and troughs of parasite abundance within hosts and yet remain poorly understood, especially when focal parasites share a host with other parasite species that are capable of modulating both "predator" (i.e., immune) behaviour and resource availability. 
We can study resource and immunological regulatory processes in parasite communities by experimentally introducing multiple parasite species into hosts, either in sequence or together (Knowles 2011, Fenton et al. 2014). Indeed, a unique advantage of within-host systems is our capacity to obtain near-replicates of ecosystem dynamics, and to manipulate hypothetical drivers experimentally so that they can be identified and quantified. Here, we use laboratory mice as hosts to parasite species with life histories that generate both resource and immunological conflicts, as follows. The welldescribed rodent malaria (Plasmodium chabaudi chabaudi clone AS) consumes red blood cells (hereafter RBCs) by invading, replicating, and then bursting out after 24h to find new target cells (Carter and Walliker 1975). The parasitic nematode Nippostrongylus brasiliensis, like several species that infect human hosts (Sarinas \& Chitkara 1997), depletes RBCs because larvae damage host tissues when migrating through the lung early in infection (Chen et al. 2012). Lung haemorrhaging reduces RBC density by approximately $5 \%$ in mice given a standard laboratory dose of $N$. brasiliensis (Hoeve et al. 2009). During anaemia, we expect the population of RBCs to shrink and to become biased towards younger RBCs (reticulocytes) as host bone marrow releases new cells to replace those lost to infection. $P$. chabaudi clone AS is among the malaria genotypes that prefers older RBCs (Paul et al. 2003). Given these life history details, we hypothesised that this nematode co-infection might limit target cell availability to malaria. 
We also hypothesised that the nematodes might facilitate malaria by modulating immunity. Adaptive immune responses of mammals fall broadly into four categories: T-helper cell type 1 (Th1), T-helper type 2 (Th2), T-helper type 17 (Th17) and T-regulatory responses (van den Ham et al. 2013). We focus on Th1/ Th2 here, because the Th1 response is essential to clearance of intracellular parasites like malaria, while Th2 is essential to clear intestinal nematodes, and because Th1 and Th2 responses are mutually inhibitory (van den Ham et al. 2013). This generates immunological conflict, potentially impairing malaria clearance in individuals co-infected with nematodes. For example, Th1-associated cytophilic antibodies, such as IgG2a in mice, block malaria development within RBCs, tag infected RBCs for destruction by phagocytes, and interfere with parasite dispersal following RBC rupture (e.g., Bergmann-Leitner et al. 2009). Nematode-induced suppression of Th1 immunity can reduce production of such antibodies and prolong malaria infection (Fenton et al. 2008).

Here, we report the first analysis that disentangles effects of resource limitation and immune facilitation upon parasite dynamics during co-infection, thereby achieving a rare identification of determinants of ecological dynamics across replicate ecosystems. We previously observed reduced peak density of malaria parasites in nematode co-infected hosts, and were unable to identify any simple immunological explanation for that outcome because peak production of the main Th1 cytokine interferon (IFN)- $\gamma$ was suppressed by the nematode (Hoeve et al. 2009). We now go beyond minima and maxima to 
measure regulation of daily parasite dynamics, and to test whether nematodeinduced anaemia (Hoeve et al. 2009; Chen et al. 2012) and/or Th1 suppression regulates malaria population size. We first examine the structure and magnitude of the immune response in infection and co-infection. We next use a metric of malaria population growth called Effective Propagation Number (Metcalf et al. 2011), which quantifies transmission of malaria from infected to uninfected cells each day (Fig. 1). Using these tools, we analyse data from three previously reported (Hoeve et al. 2009; Fairlie-Clarke et al. 2010) and four new experiments. We identify the best predictors of effective propagation and address the following hypotheses about the regulation of malaria in the presence and absence of anaemia-inducing nematodes:

1. a) Nematode co-infection will reduce the average age and population size of target cells, thereby promoting bottom-up control of malaria. b) Nematode co-infection will alter the structure and magnitude of the immune response, thereby reducing top-down control of malaria.

2. Target cell limitation will have stronger effects on malaria propagation than will immune suppression.

3. When nematode infection precedes malaria infection by a considerable period, bottom-up regulation of malaria will be diminished.

\section{Methods}

All experiments (including those generating the pathology, parasitology and univariate cytokine data reported in Hoeve et al. 2009 and the endpoint 
antibody data reported in Fairlie-Clarke et al. 2010; plus the 4 new experiments reported here) were carried out in accordance with the Animals (Scientific Procedures) Act 1986, and were approved by the UK Home Office inspectorate and institutional review committee. In total, we measured parasite, resource and immune dynamics (e.g., Figs. 2 \& 3) in 58 uninfected, 111 nematode infected, 190 malaria infected, and 121 co-infected hosts. We note that obtaining data on lung damage, nematode burden and lymph node cells requires terminal sampling, so subsets of mice were culled at early time points (see Hoeve et al. 2009), while others were observed for the entire course of acute malaria. These constraints meant that we were unable to account for variation in nematode burden in our analyses of malaria dynamics during co-infection. We instead focus on the presence/absence of nematode co-infection.

\section{1a) Simultaneous infection experiments}

We randomly assigned adult female BALB/c mice to receive standard laboratory doses of $10^{5}$ P. chabaudi chabaudi clone AS-infected RBCs and/or 200 N. brasiliensis L3 larvae. Control mice were treated with sham injections (naive RBC or saline) only. We measured the density of malaria-infected and uninfected RBCs daily, as previously described (Hoeve et al. 2009). In three experiments, randomly selected mice from each treatment group were culled at three, five, or seven days post infection (p.i.). These mice were then unavailable for daily monitoring of malaria dynamics but were invaluable in elucidating immunoparasitology of co-infection: We obtained nematode counts 
and collected cells from thoracic and mesenteric lymph nodes (to test for local differences relating to nematodes migrating through lungs and intestines, respectively). We cultured cells in RPMI media or RPMI containing T cell mitogen Concanavalin A (Con A). We measured production of immune signaling molecules, or cytokines, associated with Th1 or Th2 immune responses (interleukins (IL) IL-4, IL-5, IL-6, IL-10, IL-13, interferon (IFN)- $\gamma$, and tumour necrosis factor (TNF)- $\alpha$ ) after 72 hours of culture using cytometric bead arrays (BD Pharmingen), as previously described (Hoeve et al. 2009).

Via microscopy at 1000X magnification across at least 2 fields of view (which reproducibly comprised $\sim 1400$ RBCs per blood smear), we also counted infected and uninfected young (reticulocyte) and mature RBCs. Reticulocytes were distinguished on Giemsa-stained thin blood smears via differential nucleic acid staining (following Swardson-Olver et al. 2002). In two experiments we also measured IgG2a antibody concentration daily, as described in Supplementary Information $\S 1 \mathrm{a}$, to enable us to estimate the role of immune pressure in daily malaria dynamics.

\section{1b) Statistical analysis}

All analyses were done in $\mathrm{R}$ ( $\mathrm{R}$ Core Team 2014). Where relevant we report estimated coefficients \pm one standard error. For mixed model selection decisions, we report change in AIC. Where interactions among factors were significant, we determined pairwise differences using Tukey's Honest Significant Difference post-hoc test adjusted for multiple comparisons. 
We tested for variation in the daily densities of infected, uninfected, and young RBCs among treatment groups using repeated measures nested ANOVA, with a fixed effect of treatment (two-level factor, malaria-infected versus coinfected), and random effects of mouse nested in experiment. We analysed daily dynamics of young RBCs in terms of both their density and the percentage of uninfected RBCs that were young (i.e., \% reticulocytes). We found that the percentage metric explained greater variance among mice and better distinguished co-infected mice from those with malaria only. Subsequent analyses of malaria propagation thus focus on percentage rather than density of reticulocytes as a metric of RBC age structure.

We used ordination techniques to reveal patterns in multivariate cytokine data (Bourke et al. 2013). We looked for orthogonal linear associations that captured the greatest variance in production of the seven cytokines using Principal Components Analysis (PCA). We transformed cytokine expression data by $\log _{10}(x+1)$, and scaled the covariances to reduce skew (Jolliffe 2002, Jackson et al. 2004). We used single value decomposition in our PCA because it is computationally efficient (Jolliffe 2002), and is applicable to multiplex data where there are fewer observations (cell samples per mouse) than variables (cytokines). We followed the methods of Turner et al. (2003) and Jackson et al. (2004) to reduce dimensionality. We focused on the first three principal components (PCs1-3), which accounted for $>80 \%$ of variance in cytokine expression and had bootstrapped eigenvalues greater than one (a 
measure of their ability to explain significant variance; Jolliffe 2002).

We ran separate PCAs on data subsetted by mouse treatment (uninfected, malaria-only, nematode-only, and co-infected), by day of cull (three, five, or seven days post infection), by lymph node (mesenteric or thoracic), and by cell culture stimulus (medium and Con A). We then used ANOVA to test whether PC loadings were significantly affected by five factors: nematodeinfected/uninfected and malaria-infected/uninfected treatment factors (both two levels), day (three levels), lymph node (two levels), and cell culture stimulus (two levels). When there was no significant difference, the PCA was repeated on aggregated data starting with the factor with the smallest statistical effect (following Jackson et al. 2004). For example, although Con A stimulation significantly increased cytokine production compared to cells cultured in media alone (Hoeve et al. 2009), its effects were consistent across cytokines, treatment groups, days and lymph nodes. Cell culture stimulus was therefore collapsed out of the model.

This process established that cytokine profile only differed by treatment, so we averaged cytokine expression across media and lymph nodes to get one score per mouse, and included all mice culled on days 3,5 , and 7 in a single PCA. We modelled these PCA scores for PCs1-3 and days 3, 5, and 7 with mixed-effects ANOVA. The model included nematode-infected/uninfected and malaria-infected/uninfected fixed factors, plus a random factor for experiment (two levels). 
In each generation, infected RBCs arise from an encounter between infected $\left(I_{t}\right)$ and uninfected (susceptible) cells $\left(S_{t}\right)$, and a transmission rate (here denoted $\left.P_{e, t}\right)$ that can be described using the classic epidemiological relationship:

$I_{t+1}=P_{e, t} I_{t} S_{t}$ Eqn 1

For a schematic of this logic, see Fig. 1. Linearising this equation by taking the log allows us to calculate effective propagation, $P_{e, t}$ at every time-step for each mouse (Metcalf et al. 2011). We partitioned the degree to which $P_{e, t}$ variation was explained by $\mathrm{RBC}$ age profile (\% reticulocytes) and by immunity (IgG2a concentration), using a linear mixed effects model. In other words, our response variable was daily $P_{e, t}$. We fitted coefficients for fixed effects of antibody concentration, percentage of RBCs that were reticulocytes, presence or absence of $\mathrm{Nb}$ co-infection (binary factor, given that we could not obtain nematode burden data from mice in which we observed longitudinal malaria dynamics), and time (9 level factor: days 7 to 15 post infection). We also fitted all 2- and 3-way interactions among antibody, reticulocytes and treatment.

We ensured that the response variable conformed with parametric assumptions of linear regression and scaled fixed effect variables so that model coefficients for antibody and RBC profile could be directly compared. We excluded three IgG2a observations for which scaled antibody 
concentration was $>5$ times the standard deviation above the mean. To account for daily measures on individual mice, we included random effects of mouse and experiment. The saturated model (with all 2- and 3-way interactions among the factors denoted by *) was: $Y_{i j t}=Y_{00}+Y_{01}\left(\text { antibody }_{i j t}\right)^{*} \gamma_{02}\left(\right.$ reticulocytes $\left._{i j t}\right){ }^{*} \gamma_{03}\left(\right.$ treatment $\left._{i}\right)+\gamma_{04}(t)+u_{0 i}+$ $u_{1 j}\left(\right.$ experiment $\left.{ }_{i j}\right)+r_{i j t}$ Eqn 2 where $\boldsymbol{y}$ is a fixed cofficient, $\boldsymbol{u}$ is a random coefficient, $\boldsymbol{i}$ is individual mouse (69 level factor), $\boldsymbol{j}$ is experimental replicate (2 level factor), and $\boldsymbol{t}$ is time (9 level factor). For fixed effects, we followed the model selection method of Zuur et al. (2009) by preferring the minimal AIC. We report model fit using REML with Imer in the Ime4 package (Bates et al. 2014) and marginal and conditional $R^{2}$ (Nakagawa and Schielzeth 2013).

\section{2a) Sequential infection experiments}

To further investigate whether nematode-induced anaemia or antibody responses were more influential in determining malaria population dynamics, we shifted the timing so that nematode-induced anaemia was resolved prior to malaria infection, but nematode-induced reduction in Th1 immunity remained. Mice were infected with 200 nematode L3 and 35 days later with $10^{5}$ clone AS malaria-infected RBCs. Sequentially infected mice are hereafter denoted as nematode-then-malaria infected (as distinct from simultaneously co-infected mice). Malaria dynamics were measured as for simultaneous co-infection. Malaria-specific IgG2a was measured by ELISA in plasma samples taken at the end of infection (day 20 post-malaria infection) to ensure nematode- 
induced changes in antibody were comparable to the simultaneous coinfection regime. Details of these methods are described in Supplementary Information §1b.

\section{2b) Statistical analysis}

We tested for differences between "nematode-then-malaria" mice and malaria-only mice in effective propagation (calculated as above) and other RBC dynamics using mixed effects ANOVA. We had two fixed factors of treatment (two-level factor: with and without nematode) and day (nine level factor), and random effects of mouse ( $n=60)$ and experiment (2 levels). We ran similar models but dropped the effect of day when analysing day $20 \lg G 2 a$ antibody titre and minimum RBCs.

\section{Results}

Hypothesis 1a) Nematode co-infection will reduce the average age and population size of target cells, thereby promoting bottom-up control of malaria.

Nematode infection did alter RBC dynamics. Consistent with our previous report on a subset of these mice (Hoeve et al. 2009), nematode treatment was associated with reduced density of malaria-infected RBCs, around the time of peak density (Fig. $2 \mathrm{~A}, \beta=-0.13$, se $=0.04, F_{1,430}=7.66, p<0.01$ ). Further analyses indicated that nematode co-infection slowed malaria replication (coinfected mice had delayed parasitemia, Fig. S1A), and produced delayed (Fig. S1B) and milder anaemia (Fig. S2). Most important for our hypothesis about 
bottom-up control, co-infected mice had more uninfected and young RBCs than malaria-only mice in the second week of infection (Fig. 2B, nematode treatment factor in mixed effects model of infection metrics from day 8 to 15; uninfected RBCs $\beta=0.63$, se $=0.18, F_{1,347}=14.4, p<0.01$; reticulocytes $\beta=0.02$, se $\left.=0.02, F_{1,143}=4.06, p<0.05\right)$. These results accord with the hypothesised effect of nematodes on malaria resources.

Hypothesis 1b) Nematode co-infection will alter the structure and magnitude of the immune response, thereby reducing top-down control of malaria.

Nematode infection also altered the structure and magnitude of the developing immune response. We found that cytokine expression across all mice could be adequately described by a single PCA of three components (see Methods). We examined the loadings of cytokines on the first three PCs (Fig. 3A-C) to ascertain the structure of the cytokine network. PC1 represented the overall magnitude of immune response, being positively correlated with all cytokines (Fig. 3A, 34\% variance), PC2 represented a Th1/Th2 axis with IFN- $\gamma$ and TNF- $\alpha$ opposed to the other five more Th2associated cytokines (Fig. 3B, 19\% variance), and PC3 was driven by the cytokine IL-6, which has unique roles in inflammation and tissue repair (Fig. 3C, $15 \%$ variance, Fernando et al. 2014). These interpretations were robust in subsets of the data (e.g., within treatment groups).

We also examined how the scores of mice differed by treatment on the first 
three PC axes (Fig. 3D-F). On PC1, nematode-infected mice had lower scores than nematode-uninfected mice, consistent with nematodes being immunosuppressive (Fig. 3D nematode $F_{1,158}=301.7 \mathrm{p}<0.001$, malaria $F_{1,158}=19.4 \mathrm{p}<0.001$, interaction $\left.F_{1,158}=1.3 \mathrm{p}>0.2\right)$. On PC2, nematode-infected mice had higher expression consistent with Th2-biased cytokine responses, while malaria-infected mice had lower expression consistent with Th1-biased responses (Fig. 3E, nematode $F_{1,158}=17.8 p<0.001$, malaria $F_{1,158}=73.2$ $\mathrm{p}<0.001$, interaction $\left.F_{1,158}=2.2 \mathrm{p}>0.1\right)$. On PC3, nematode-only mice had the highest expression, marginally lessened by malaria co-infection, with malariaonly mice lower still and uninfected mice the lowest (Fig. 3F, nematode $F_{1,158}=80.8 p<0.001$, malaria $F_{1,158}=6.7 p<0.05$, interaction $F_{1,158}=5.3 p<0.05$ with Tukey $p>0.14$ for nematode-infected versus co-infected mice). These results accord with hypothesised effects of nematodes on the type of immune response needed to clear malaria and provide broad cytokine-level support for our previous observation that nematode infection reduces IgG2a antibody titres in these mice (Fairlie-Clarke et al 2010). However, none of these findings help to explain why co-infected mice had lower, rather than higher, malaria parasite burdens than mice with malaria only.

Hypothesis 2) Target cell limitation will have stronger effects on malaria propagation than will immune suppression.

As hypothesised, nematodes affected both the RBC and cytokine profiles, so we proceeded to study associations between resource availability (\% young 
RBCs), the effector immune response (Th1-driven IgG2a antibody), and malaria effective propagation. Given our previous meta-analytic findings that bottom-up control is more potent than top-down control of co-infection in both mice (Graham 2008) and people (Griffiths et al. 2014), we predicted that the effect of nematodes on RBCs would be the dominant mechanism regulating malaria within co-infected hosts.

Indeed, the final model for effective propagation included young RBCs and day of observation (Table 1 , marginal $R^{2} 0.321$, conditional $R^{2} 0.407$ ). The higher the proportion of young RBCs, the lower the effective propagation (Fig.4, fitted coefficient $\beta=-0.1$, Table 1). This negative coefficient indicates resource limitation. The significant factor of day post infection indicated consistent daily dynamics across mice in effective propagation ( $F>10$, Table 1). Neither the main effect of IgG2a antibody nor of treatment per se had a significant association with effective propagation. Even when antibody was included in the model, its coefficient was less than a tenth of that of young RBCs ( $\beta$ being comparable because the variables were scaled). A borderline term in the model was the young RBC-by-treatment interaction, which increased AIC by less than 1, though its standard error was almost equal to its effect size (Table 1; compare circles and triangles in Fig. 4).

We found additional evidence that resource depletion suppressed malaria because the higher the proportion of young RBCs the fewer the infected cells (Fig. S3). We thus found evidence for resource depletion of malaria by 
nematode co-infection, but limited evidence for IgG2a antibody affecting malaria dynamics.

Hypothesis 3: When nematode infection precedes malaria infection by a considerable period of time, bottom-up regulation of malaria will be diminished.

Finally, we ran experiments to remove the coincidence of malaria infection and nematode-induced anaemia and to test for an effect of IgG2a antibody on malaria. As hypothesised, when nematode-induced anaemia preceded Pccinfection by 5 weeks, there was no difference between nematode-then-malaria and malaria-only treatments in infected or uninfected RBC density $(\beta=0.01$, se $=0.04, F_{1,51}=0.17, p>0.68$, Fig. $\left.5 \mathrm{~A}, \mathrm{~B}\right)$ or effective propagation $(\beta=1.5$, $\left.\mathrm{se}=8.7, F_{1,51}=0.02 \mathrm{p}>0.8\right)$.

Nematode-infected mice had reduced malaria-specific lgG2a antibody titre on day 20 compared with malaria-only mice (Fig. 5C), as during simultaneous infection (Fairlie-Clarke et al. 2010). This treatment effect on antibody did not translate into any detectable difference in malaria dynamics, which corroborates our finding under Hypothesis 2 in simultaneous co-infection that RBC age structure affects malaria population dynamics more than immunity.

\section{Discussion}

Here, we show that both the red blood cells consumed by malaria parasites and immune responses to malaria were suppressed by a lung-migratory 
nematode. We found that only the bottom-up mechanism was consistent with malaria propagation during co-infection. The study is rare in simultaneously measuring both resources and immunity, and then using an epidemiological framework to model within-host dynamics. We suggest that considering immune and resource-mediated mechanisms of parasite regulation in tandem is essential to understand the dynamics of co-infections, even in the laboratory. We further suggest that, in the field, resource-mediated mechanisms could be as important as immunity in determining effects of helminths on malaria incidence and severity. More generally, our findings demonstrate the utility of near-replicate experimental "mesocosms" in identifying and quantifying drivers of population dynamics. While hosts, even inbred laboratory mice, cannot provide perfectly replicated ecosystems, they do come close, and enable researchers to leverage that variation (e.g., subtle differences among hosts in starting RBC density) to explain dynamics.

We hypothesised that nematode infection would reduce the average age and population size of RBCs, due to haemorrhaging during migration through the lung (Chen et al. 2012). We indeed found that nematode-infected mice had a higher frequency of young RBCs than mice with malaria only. Co-infected also mice experienced delayed malaria dynamics, with potential implications for chronicity and thus transmission as in other malaria-helminth systems (e.g., Noland et al. 2007). Younger RBC age profiles were associated with reduced malaria propagation. We attribute this to a biological preference of this genotype of malaria parasites to replicate within mature RBCs (Paul et al. 
2003). This tendency not to invade young RBCs has previously been modelled for this malaria clone in T-cell intact and depleted mice (Mideo et al. 2008). Our analyses now show how target cell availability also regulated malaria population size in immunocompetent mice co-infected with nematodes. The better fits for frequency rather than density of young RBCs is suggestive of a frequency-dependent transmission process - i.e., the frequency of target cells may determine how many new infections are established by a single infected RBC. This implies that spatial structure within the blood may be shaping access of merozoites to new cells, an interesting area for future work especially now that in vivo behaviour of cells can be tracked.

We also hypothesised that nematodes would suppress immune responses against malaria, and we did find support for this prediction. Furthermore, we found a stable structure to relationships among immune signaling molecules across hosts of differing infection status. In other words, accounting for variation among mice in overall magnitude of cytokine responses (our PC1), elevated Th2 cytokines predictably were associated with reduced Th1 cytokines (our PC2). These findings concur with other studies of helminth coinfection (e.g., Jackson et al. 2004). Production of the Th1-associated antibody (IgG2a) needed for malaria clearance was also suppressed following simultaneous (Fairlie-Clarke et al. 2010) and sequential nematode infection. However, we report here that immunosuppressive effects of nematode coinfection were unimportant to malaria propagation. 
Our sequential infection results further suggest that target cell availability exerts a stronger impact on malaria dynamics than antibody. When nematode-induced anaemia was resolved prior to malaria infection, malaria density and effective propagation were similar in single and sequentially infected mice despite IgG2a suppression. This represents a step toward experimentally untangling resource versus immune-mediated mechanisms of control. However, in this design, the timing of nematode-induced innate immune responses were also altered relative to malaria infection, which may influence malaria dynamics. Previous malaria modeling work suggested innate immunity regulated early malaria dynamics, with some involvement of resources (Kochin et al. 2010), though others found that resource limitation captures the main features of malaria dynamics (Antia et al. 2008, Metcalf et al. 2012). Indeed, whilst we argue that resource availability is more influential than immune response in regulating malaria dynamics, we acknowledge that these processes are not mutually exclusive. For example, 'bystander killing' of uninfected RBC by phagocytes (Tippett et al. 2007) is an immune-mediated mechanism that reduces availability of RBCs; also, nematode migration might induce innate responses (Chen et al 2012) that promote killing of malaria parasites. Further experiments are warranted, not least to explore dosedependence in the effects of nematodes on malaria propagation.

Our findings may be relevant to variation in the course of malaria in humans. Malaria is of major global health concern and often occurs in areas with endemic helminth infections (Righetti et al. 2012). Human malarias exhibit 
differential preferences for young versus mature RBCs (Paul et al. 2003). If nematodes infecting humans chronically skew RBC age profiles through haemorraging and, in the case of hookworm, direct consumption of RBCs, subsequent malaria dynamics, including recrudescence, might be altered. For example, concurrent hookworm and RBC-generalist $P$. falciparum infections do result in more severe malarial anaemia in preschool children (Brooker et al. 2007). By contrast, we would predict ameliorated anaemia if hookworm cooccurred with mature-RBC-specialist $P$. malariae. We are unaware of any study that has yet investigated such co-infection. It is important to note that resource-mediated interactions between helminths and malaria may in some contexts be mediated by RBC availability per se, rather than RBC age structure, or even by iron deficiency. Indeed, iron supplementation feeds $P$. falciparum, to the detriment of the host (Clark et al. 2014). If continued research generates strong support for resource-mediated interactions between human malaria and helminths, then medical interventions such as anthelmintics, antimalarials, and erythropoietin (Chang \& Stevenson, 2004) may need to be selected and paired according to which parasites co-occur in local hosts.

Beyond malaria, our findings confirm predictions arising from our prior metaanalyses of co-infected laboratory mice (Graham 2008) and co-infected people (Griffiths et al. 2014) that suggested a more prominent role for bottomup than top-down processes regulating a diverse array of parasite populations in co-infected hosts. Likewise, experiments in wild mice in which within-host parasite communities were perturbed by anthelminthic treatment revealed 
interactions between nematodes and protozoa that share infection sites, suggesting local, likely resource-mediated competition (Knowles et al. 2013, Pedersen and Antonovics 2014). However, our findings differ from simian immunodeficiency virus for which target cell limitation has weaker effects than immunity on ecological dynamics (Regoes et al. 2004). We urge further studies incorporating parasite propagation, resources, and immune metrics to gauge the distribution of infections and co-infections for which bottom-up regulation exceeds top down regulation.

In addition, we suggest that our quantitative approach has the potential to provide (perhaps surprising) general insights into between-host epidemiology and immunology. The core principles that drive measles dynamics across populations echo those of within-host malaria dynamics (Metcalf et al. 2011): susceptible individuals (for the measles case) or RBCs (for malaria) are infected at a time-varying rate, and then either recover (for measles) or die (for malaria). The unique power of within-host mouse malaria is that with dynamics driven by the same basic principles as measles or other childhood infection systems (Susceptible-Infected-Recovered (SIR)-like dynamics), we can use experiments to identify and quantify the empirical details of further drivers, including immune system activity per se, independent of resources.

More generally, despite the closed nature of the mammal-as-ecosystem in which immune "predators" and bodily resources are so intertwined (Pederson \& Fenton 2007), we offer our results as an example of experimental 
identification of the ecological drivers of population dynamics. The free-living analogy of our work would be introduction into a grassland of a new herbivore species that simultaneously alters predator behaviour and resource availability to a focal species, followed by the demonstration that changed resources determine the subsequent dynamics of the focal species. Such an approach complements the longitudinal, observational approaches that have quantified how competition (e.g., Coulson et al. 2001; Jacobson et al. 2004; Martorell and Freckleton 2014) or predation (e.g., O'Donoghue et al. 1998) drive population dynamics in the field. It will be fascinating to learn whether bottom-up processes outweigh top-down in importance across systems. Experimental approaches could be used to investigate ecological processes in other tritrophic systems - e.g., to explore food web dynamics in replicate mesocosms, in lab or field, and to investigate relative impacts of bottom-up and top-down processes. Indeed, recent experiments revealing cascading effects across trophic scales (e.g., parasites reducing availability of prey for predators; Banerji et al. 2015) may represent a crucial frontier in functional ecology in which interactions or temporal synergies among resources, parasites and predators can be dissected across food webs. We keenly await more such work.

Acknowledgements

We acknowledge the invaluable contributions of the animal care staff at the University of Edinburgh, Tina Hansen for microscopy, Kathryn Watt and 
Sheelagh Duncan for technical assistance, and Rick Maizels and Yvonne Harcus for the nematodes. We thank David Dunne, Jan Bradley, Sarah Reece, Anieke van Leeuwen and Sarah Budischak for comments on previous drafts of this work. We thank Neil Rooney, Owen Petchey, Amy Pedersen, and Andy Fenton for discussion and Gustavo Henrique de Carvalho for statistical advice. We are also very grateful to our funders. The empirical work was funded by the U.K. Biotechnology and Biological Sciences Research Council (grant BB/C508734/1 to JEA and fellowship BB/D01977X/1 to ALG). Santander Bank Universities Scheme Research Mobility Award via the University of Sheffield funded ECG's visit to work with Gustavo at Universidade Federal de Sao Carlos, Brazil. ECG's visit to Princeton was funded by a BES small project grant, BSP international travel award, and a Princeton University Visiting Student Research Collaborator award.

\section{References}

1.

Andrewartha, H. G. \& L. C. Birch (1954). The Distribution and Abundance of Animals. Chicago, University of Chicago Press.

2.

Antia, R., Yates, A., \& de Roode, J. C. (2008). The dynamics of acute malaria infections. I. Effect of the parasite's red blood cell preference. Proc Roy Soc B, $275 ; 1449-1458$.

3.

Banerji, A., A. B. Duncan, J. S. Griffin, S. Humphries, O. L. Petchey \& O. Kaltz 
(2015). Density- and trait-mediated effects of a parasite and a predator in a tri-trophic food web. J Anim Ecol 84: 723-733.

4.

Bates, D., Bolker, B., Maechler, M., \& Walker, S. (2014). Linear mixed-effects models using Eigen and S4. R package version 1.1-7. Available at: http://CRAN.R-project.org/package=Ime4

5.

Bergmann-Leitner, E.S., Duncan, E.H., \& Angov, E. (2009). MSP-1p42specific antibodies impact growth and development of intra-erythrocytic parasites of Plasmodium falciparum. Malar J 8; 183.

6.

Bourke, C. D., Nausch, N., Rujeni, N., Appleby, L. J., Mitchell, K. M., Midzi, N., et al. (2013). Integrated analysis of innate, Th1, Th2, Th17, and regulatory cytokines identifies changes in immune polarisation following treatment of human schistosomiasis. J Inf Dis, 208; 159-169.

7.

Brooker, S., W. Akhwale, R. Pullan, B. Estambale, S. E. Clarke, R. W. Snow \& P. J. Hotez (2007). Epidemiology of plasmodium-helminth co-infection in Africa: populations at risk, potential impact on anemia, and prospects for combining control. Am J Trop Med Hyg 77: 88-98.

8.

Carter, R., \& Walliker, D. (1975). New observations on the malaria parasites of rodents of the Central African Republic-Plasmodium vinckei petteri subsp. nov. and Plasmodium chabaudi Landau, 1965. Ann Tropical Med Parasitol, 
69; 187-196.

9.

Chang, K.H., \& Stevenson, M.M. (2004). Malarial anaemia: mechanisms and implications of insufficient erythropoiesis during blood-stage malaria. Int $J$ Parasitol, 34; $1501-1516$.

10.

Chen, F., Z. G. Liu, W. H. Wu, C. Rozo, S. Bowdridge, A. Millman, N. Van Rooijen, J. F. Urban, T. A. Wynn \& W. C. Gause (2012). An essential role for T(H)2-type responses in limiting acute tissue damage during experimental helminth infection. Nat Med 18: 260-266.

11.

Clark, M. A., M. M. Goheen, A. Fulford, A. M. Prentice, M. A. Elnagheeb, J. Patel, N. Fisher, S. M. Taylor, R. S. Kasthuri and C. Cerami (2014). Host iron status and iron supplementation mediate susceptibility to erythrocytic stage Plasmodium falciparum. Nat Comm 5: 4446.

12.

Cornulier, T., N. G. Yoccoz, V. Bretagnolle, J. E. Brommer, A. Butet, F. Ecke, D. A. Elston, E. Framstad, H. Henttonen, B. Hornfeldt, O. Huitu, C. Imholt, R. A. Ims, J. Jacob, B. Jedrzejewska, A. Millon, S. J. Petty, H. Pietiainen, E. Tkadlec, K. Zub \& X. Lambin (2013). Europe-Wide Dampening of Population Cycles in Keystone Herbivores. Science 340(6128): 63-66. 13.

Coulson, T., E. A. Catchpole, S. D. Albon, B. J. Morgan, J. M. Pemberton, T. H. Clutton-Brock, M. J. Crawley \& B. T. Grenfell (2001). Age, sex, density, winter 
weather, and population crashes in Soay sheep. Science 292: 1528-1531. 14.

Fairlie-Clarke, K.J., Lamb, T.J., Langhorne, J., Graham, A.L., \& Allen, J.E. (2010). Antibody isotype analysis of malaria-nematode co-infection: problems and solutions associated with cross-reactivity. BMC Immunol, 11; 6 .

15.

Fenton, A., T.J. Lamb \& A.L. Graham (2008). Optimality analysis of Th1-Th2 immune responses during microparasite-macroparasite co-infection, with epidemiological feedbacks. Parasitol 135; 841-853.

16.

Fenton, A., Pedersen, A.B. \& Petchey, O.L. (2014). The reliability of observational approaches for detecting interspecific parasite interactions: comparison with experimental results. Int J Parasitol 44; 437-445.

17.

Fernando, M.R., Reyes, J.L., lannuzzi, J., Leung, G., \& McKay, D.M. (2014). The pro-inflammatory cytokine, IL-6, enhances polarization of alternatively activated macrophages. PLOS ONE 9; e94188

18.

Graham, A.L. (2008). Ecological rules governing helminth-microparasite coinfection. Proc Nat Acad Sci USA, 105; 566-570.

19.

Griffiths, E.C., Pedersen, A.B., Fenton, A., \& Petchey, O.L. (2014). Analysis of a summary network of co-infection in humans reveals that parasites interact most via shared resources. Proc Roy Soc B. 281; 20132286. 
20.

Haydon, D.T., Matthews, L., Timms, R., \& Colegrave, N. (2003). Top-down or bottom-up regulation of intra-host blood-stage malaria: do malaria parasites most resemble the dynamics of prey or predator? Proc Roy Soc B. 270; 289298.

21.

Hoeve, M.A., Mylonas, K.J., Fairlie-Clarke, K.J., Mahajan, S.M., Allen, J.E., \& Graham, A.L. (2009). Plasmodium chabaudi limits early Nippostrongylus brasiliensis-induced pulmonary immune activation and Th2 polarization in coinfected mice. BMC Immunol, 10; 60.

22.

Jackson, J.A., Turner, J.D., Rentoul, L., Faulkner, H., Behnke, J.M., Hoyle, M., et al. (2004). T helper cell type 2 responsiveness predicts future susceptibility to gastrointestinal nematodes in humans. J Inf Dis, 190; 1804-1811.

23.

Jacobson, A. R., A. Provenzale, A. Von Hardenberg, B. Bassano and M. Festa Bianchet (2004). Climate forcing and density dependence in a mountain ungulate population. Ecology 85(6): 1598-1610.

24.

Jolliffe, I. (2002). Principal component analysis. John Wiley \& Sons, Ltd.

25.

Knowles, S.C. (2011). The effect of helminth co-infection on malaria in mice: a meta-analysis. Int J Parasitol, 41; 1041-1051.

26. 
Knowles, S.C., Fenton, A., Petchey, O.L., Jones, T.R., Barber, R., \& Pedersen, A.B. (2013). Stability of within-host-parasite communities in a wild mammal system. Proc Roy Soc B, 280; 20130598.

27.

Martorell, C., \& Freckleton, R.P. (2014). Testing the roles of competition, facilitation and stochasticity on community structure in a species-rich assemblage. J Ecol, 102; 74-85.

28.

Metcalf, C.J.E., Graham, A.L., Huijben, S., Barclay, V.C., Long, G.H., Grenfell, B.T., et al. (2011). Partitioning regulatory mechanisms of within-host malaria dynamics using the effective propagation number. Science, 333; 984-988. 29.

Metcalf, C.J.E., Long, G.H., Mideo, N., Forester, J.D., Bjørnstad, O.N., \& Graham, A.L. (2012). Revealing mechanisms underlying variation in malaria virulence: effective propagation and host control of uninfected red blood cell supply. J Roy Soc Interface, 9; 2804-2813.

30.

Mideo, N., Barclay, V.C., Chan, B.H., Savill, N.J., Read, A.F., \& Day, T. (2008). Understanding and predicting strain-specific patterns of pathogenesis in the rodent malaria Plasmodium chabaudi. Am Nat, 172; E214-E238.

31.

Nakagawa, S., \& Schielzeth, H. (2013). A general and simple method for obtaining R2 from generalized linear mixed-effects models. Methods Ecol Evol, 4; 133-142. 
32.

Noland, G. S., T. K. Graczyk, B. Fried \& N. Kumar (2007). Enhanced malaria parasite transmission from helminth co-infected mice. Am J Trop Med Hyg 76(6): 1052-1056.

33.

O'Donoghue, M., S. Boutin, C. J. Krebs, G. Zuleta, D. L. Murray \& E. J. Hofer (1998). Functional responses of coyotes and lynx to the snowshoe hare cycle.

Ecology 79: 1193-1208.

34.

Paul, R.E.L., Ariey, F., \& Robert, V. (2003). The evolutionary ecology of Plasmodium. Ecol Lett, 6; 866-880.

35.

Pedersen, A.B., \& Fenton, A. (2007). Emphasizing the ecology in parasite community ecology. Trends Ecol Evol, 22; 133-139.

36.

Pedersen, A.B., \& Antonovics, J. (2014). Anthelmintic treatment alters the parasite community in a wild mouse host. Biol Lett 9; 20130205 37.

R Core Team (2014). R: A language and environment for statistical computing. R Foundation for Statistical Computing, Vienna, Austria. Available at: http://www.R-project.org/. 38.

Regoes, R.R., Antia, R., Garber, D.A., Silvestri, G., Feinberg, M.B., \& Staprans, S.I. (2004). Roles of target cells and virus-specific cellular immunity 
in primary simian immunodeficiency virus infection. J Virol, 78; 4866-4875.

39.

Righetti, A.A., Glinz, D., Adiossan, L.G., Koua, A.Y.G., Niamké, S., Hurrell, R.

F., et al. (2012). Interactions and potential implications of Plasmodium

falciparum-hookworm co-infection in different age groups in south-central

Côte d'Ivoire. PLoS Negl Trop Dis, 6; e1889.

40.

Sarinas, P.S., Chitkara, R.K. (1997). Ascariasis and hookworm. Semin Respir Infect 12; 130-137.

41.

Singman, H., Bolker,B., Westfall,J., Hojsgaard, S., Fox,J., Lawrence, M.A., et al. (2015). R package for estimation of $p$-values from mixed model version 0.13-145. Available at: http://www.psychologie.unifreiburg.de/Members/singmann/R/afex

42.

Swardson-Olver, C.J., Dawson, T.C., Burnett, R.C., Peiper, S.C., Maeda, N., \& Avery, A.C. (2002). Plasmodium yoelii uses the murine Duffy antigen receptor for chemokines as a receptor for normocyte invasion and an alternative receptor for reticulocyte invasion. Blood, 99; 2677-2684. 43.

Tippett, E., Fernandes, L.A., Rogerson, S.J., \& Jaworowski, A. (2007). A novel flow cytometric phagocytosis assay of malaria-infected erythrocytes. $J$ Immunol Methods 325; 42-50.

44. 
Turner, J.D., Faulkner, H., Kamgno, J., Cormont, F., Van Snick, J., Else, K.J., et al. (2003). Th2 cytokines are associated with reduced worm burdens in a human intestinal helminth infection. $J$ Inf Dis, 188; 1768-1775.

45.

van den Ham, H.-J., A. C. Andeweg and R. J. de Boer (2013). Induction of appropriate Th cell phenotypes: Cellular decision-making in a heterogeneous environment. Parasite Immunol. 35; 318-330.

46.

Zuur, A.F., leno, E.N., Walker, N.J., Saveliev, A.A., \& Smith, G.M. (2009). Mixed effects modelling for nested data. In Mixed effects models and extensions in ecology with $R$ (pp. 101-142). Springer New York. 


\section{Table Legend}

Table 1. Predictor variables considered in model of effective propagation of $P c c$ in simultaneous co-infection experiments. Antibody is the scaled IgG2a concentration ( $\mu \mathrm{g} / \mathrm{mL}$ of blood). Treatment is presence/absence of nematode co-infection. The coefficient for young RBCs (scaled \%) corresponds to the final model, while other coefficients relate to the addition of that variable to the final model. P-values were estimated for each fixed coefficient in the saturated model using parametric bootstrapping with 1000 simulated datasets using the $R$ function afex::mixed (Singman et al. 2014). $\triangle A I C$ denotes the increase in this criterion with reversal of the decision to include or exclude that variable from the final model.

\begin{tabular}{lrrrrrr}
\hline & $\begin{array}{r}\text { Fitted } \\
\text { Variable }\end{array}$ & $\begin{array}{r}\text { Standard } \\
\text { error }\end{array}$ & $F$ & $p$ & Decision & $\begin{array}{l}\Delta \text { AIC if } \\
\text { decision } \\
\text { reversed }\end{array}$ \\
\hline Young RBCs & -0.104 & 0.022 & 185.30 & $<0.01$ & Keep & 90.03 \\
Day post infection & varied by day & varied by day & 10.09 & $<0.01$ & Keep & 88.19 \\
Treatment & 0.019 & 0.030 & 0.19 & 0.4 & Delete & 0.87 \\
Young RBCs:Treatment & 0.032 & 0.029 & 1.131 & 0.2 & Delete & 1.62 \\
Antibody & 0.009 & 0.022 & 9.56 & 0.68 & Delete & 1.84 \\
Young RBCs:Antibody & 0.003 & 0.016 & 0.04 & 0.70 & Delete & 1.95 \\
Antibody:Treatment & 0.027 & 0.034 & 0.33 & 0.68 & Delete & 3.34 \\
Young RBCs: & & & & & & \\
Antibody:Treatment & 0.017 & 0.018 & 0.98 & 0.18 & Delete & 2.05 \\
\hline
\end{tabular}




\section{Figure Legends}

Fig. 1. The dynamic within-host malaria ecosystem. For each generation of malaria parasites, we accounted for infected cells, from which parasites burst, and uninfected cells available for invasion (grey labels and arrows) by calculating Effective Propagation Number as an indicator of the progression of malaria infection (Metcalf et al. 2011; $P_{e, t}$ in Eqn 1). We compared the relative strength of bottom-up regulation (by the availability of older, preferred RBCs) and top-down regulation (by IgG2a antibody) by assessing their impacts on effective propagation. We also assessed whether co-infection altered these regulatory processes (nematode effects not drawn for simplicity).

Fig. 2. Daily indicators of malaria density (A) RBC resources (B) and acquired Th1 immunity $(C) \pm$ one standard error around the mean for malariaonly mice (dashed line) and malaria+nematode co-infected mice (solid line) mice. Antibody concentration was measured daily in two replicate experiments, both of which have corresponding data on percentage of young RBCs (inset, B, depicting mean and range of residuals of young RBCs for coinfected mice at left; malaria-infected mice at right, after accounting for random effects of mouse, experiment and day post infection in mixed models).

Fig. 3. Results of a Principal Components Analysis of $\log (x+1)$ transformed cytokine production by cells cultured from all mice (i.e. four treatments culled three, five, or seven days post infection), averaged across stimuli, lymph nodes and time points (see Methods). We show results by infection treatment 
for the first three principal component axes: the loadings of seven cytokines $(A-C)$, and the scores for each mouse (D-F).

Fig. 4. Effective Propagation Number $\left(P_{\mathrm{et}}\right)$ of malaria in relation to the scaled percentage of RBCs that were young RBCs. Points are a daily value per mouse with co-infection as triangles and malaria-only as circles. Grey-filled symbols are predictions from the optimal model and open symbols are for observed values. A treatment ${ }^{*} y$ oung $\mathrm{RBC}$ interaction was the final term to be deleted from the model.

Fig. 5. Malaria and RBC dynamics from mice infected with malaria on day 0 who either had been infected with nematodes 35 days prior (nematode-thenmalaria, solid line), or had not (malaria-only dashed line). A) Proportion RBCs that were infected. B) Total RBC density. Inset B) Minimum RBC density including comparison with nematode-only and uninfected mice. C) Mean IgG2a antibody titres 20 days post malaria-infection for nematode-only, nematode-then-malaria, and malaria-only mice \pm standard error. Nematodethen-malaria titres were significantly different from malaria-only (Tukey $P<0.0001)$, but not nematode-only. 


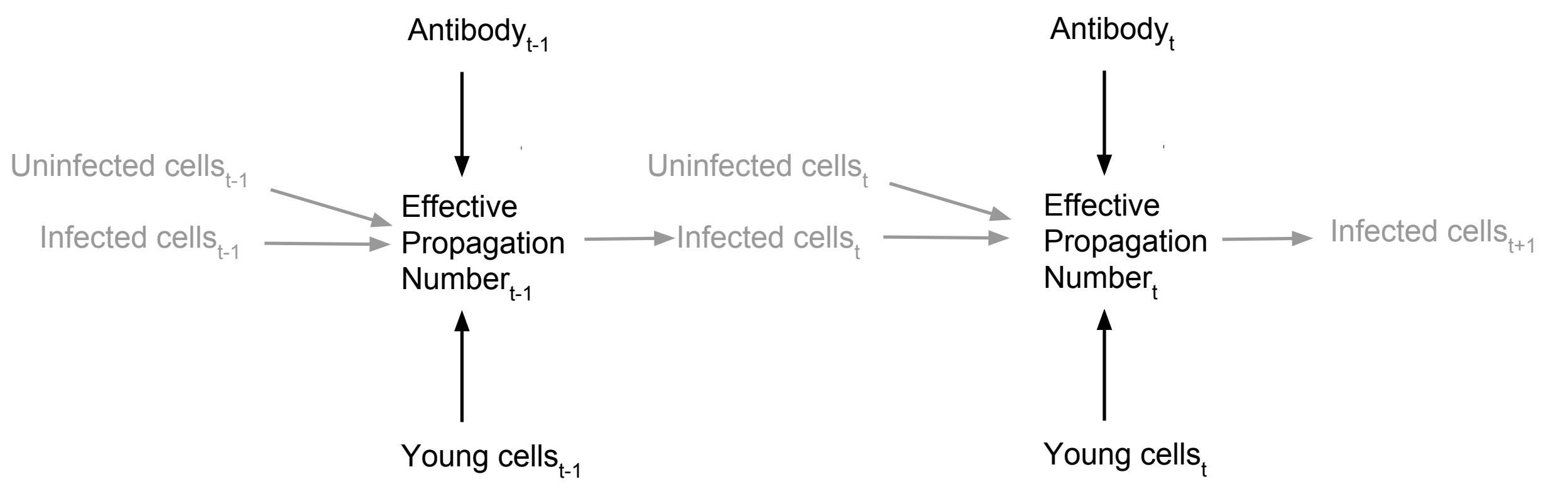



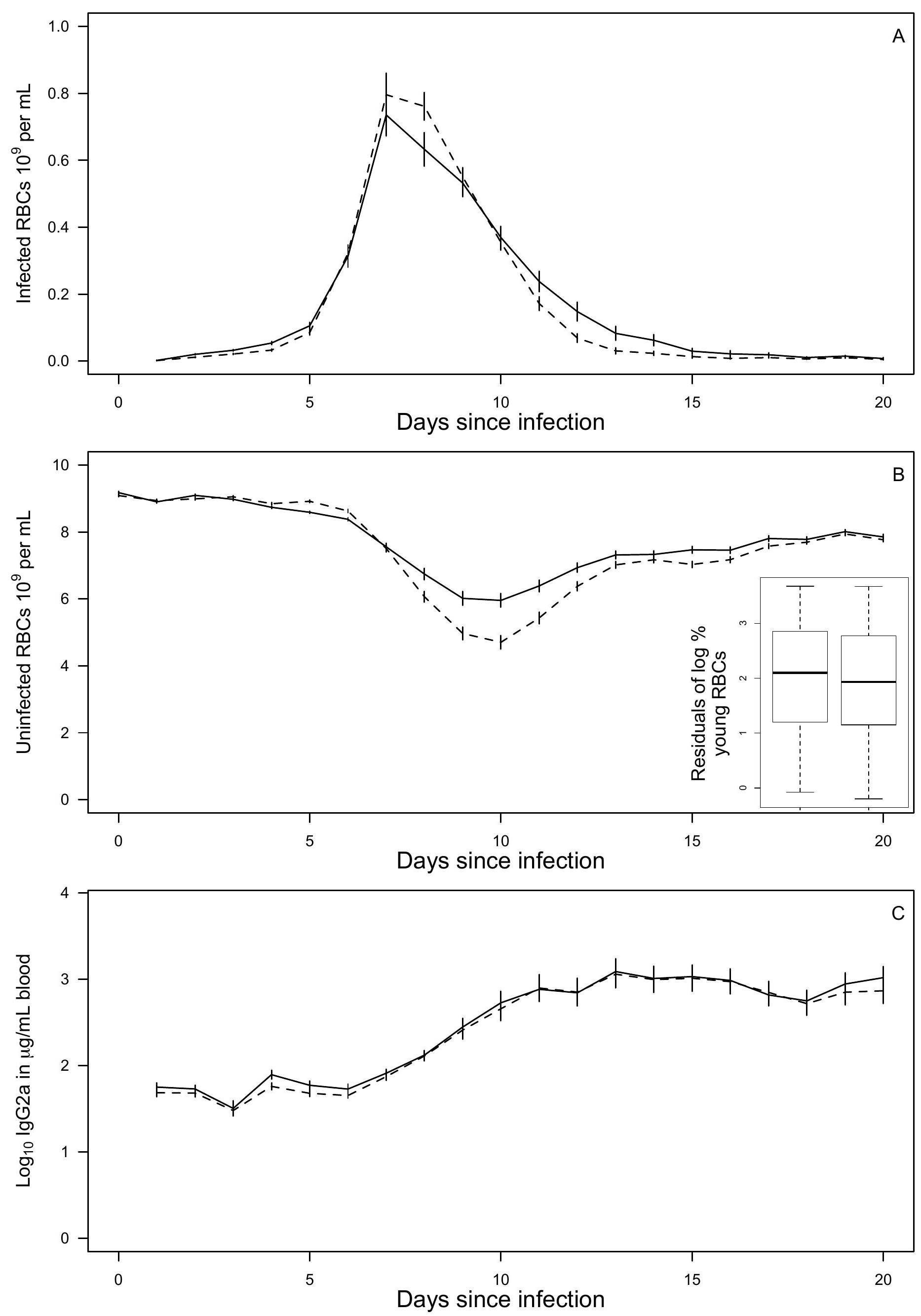


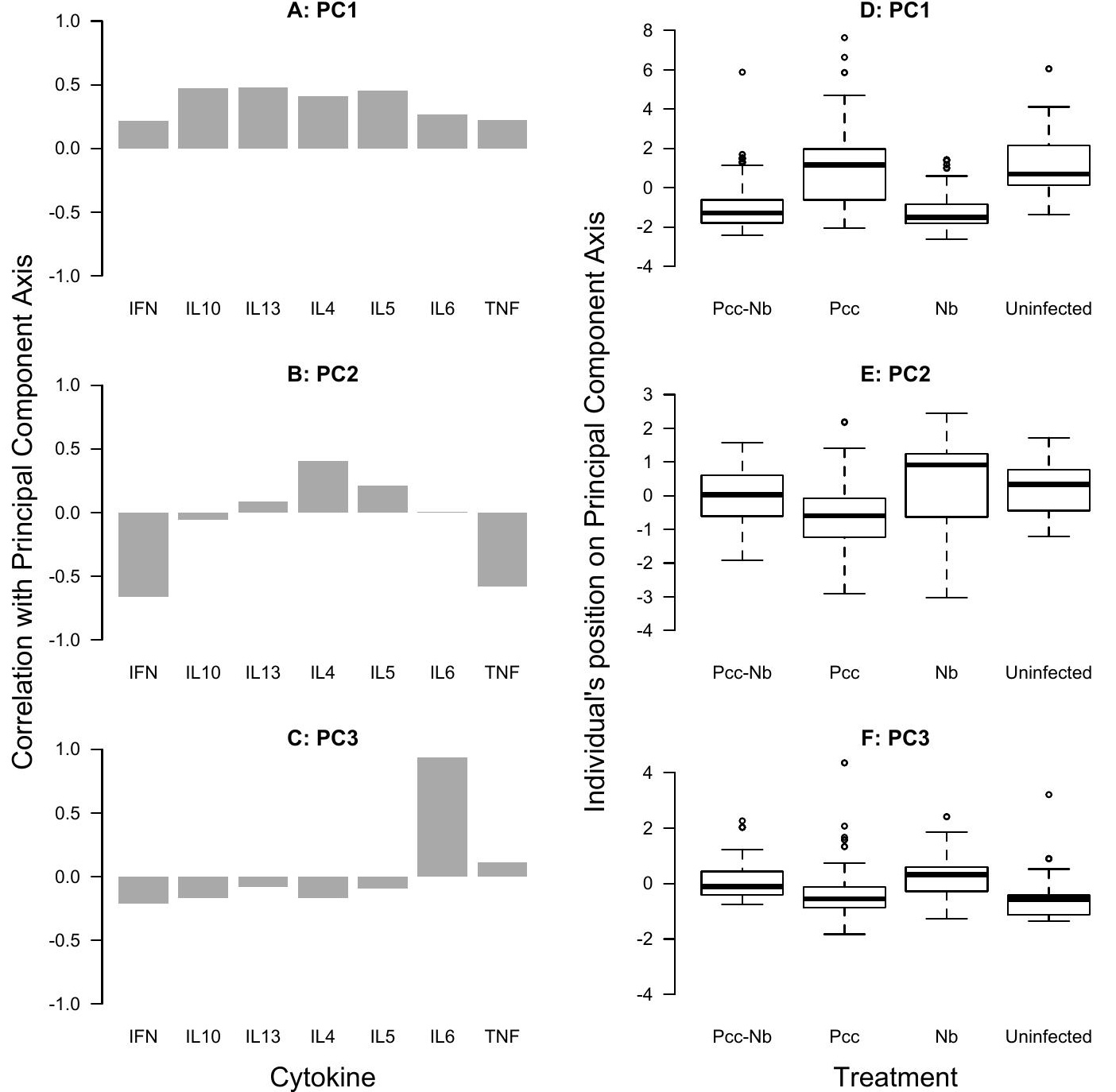




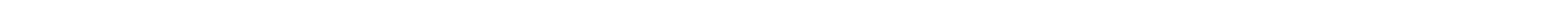



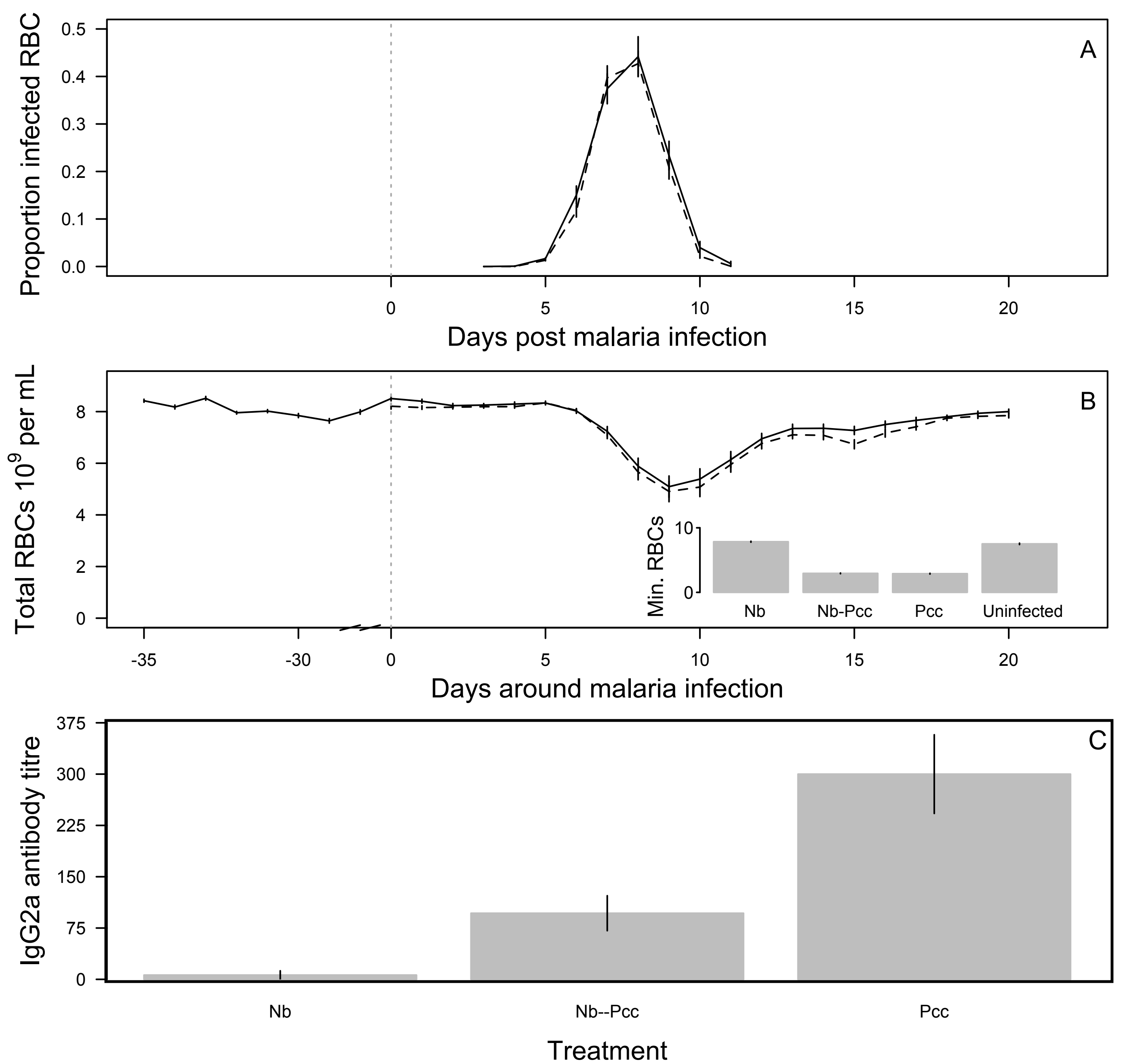